
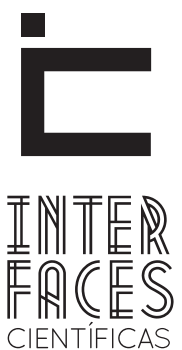

SAÚDE E AMBIENTE

\title{
PERFIL DOS PACIENTES COM NÓDULOS TIREOIDIANOS SUBMETIDOS À PUNÇÃO ASPIRATIVA POR AGULHA FINA
}

\author{
Josilda Ferreira Cruz \\ Larissa Baracho Macena ${ }^{3}$ \\ Francielle Temer de Oliveira
}

Mário Augusto Ferreira $\mathrm{Cruz}^{2}$ Priscilla Mota Coutinho ${ }^{4}$

\section{RESUMO}

O nódulo tireoidiano é a forma de apresentação de várias doenças desta glândula. É uma condição clínica muito frequente, sobretudo quando se empregam métodos sensíveis de imagem. Nesse contexto, a punção aspirativa por agulha fina (PAAF) é o principal exame utilizado para diagnóstico diferencial e o de melhor relação custo-benefício na avaliação da doença nodular da tireoide. 0 presente estudo se propõe a avaliar o perfil dos pacientes com nódulos tireoidianos que são submetidos à PAAF. A amostra consistiu em 77 pacientes que apresentavam nódulo tireoidiano a ser puncionado. Os dados foram coletados por um período de 5 meses, no Centro de Medicina Integrada de Sergipe (CEMISE), localizado em Aracaju/SE. A amostra totalizou 77 pacientes. Dentro da amostra havia 73 pacientes do sexo feminino $(94,8 \%)$ e 4 do sexo masculino (5,2\%), com faixa etária predominante entre 50 e 59 anos. Quanto ao número de nódulos, $57,1 \%$ dos pacientes (44 pacientes) tinham apenas um nódulo para ser puncionado, enquanto que $42,9 \%$ (33 pacientes) tinham mais de um. A maioria (58,3\%) dos nódulos estudados apresentou tamanho $\geq 1,0 \mathrm{~cm}$. 0 relato de história familiar de doença tireoidiana foi encontrado em 42 pacientes $(54,5 \%)$ e a história pessoal de hipotireoidismo correspondeu a $16,9 \%$ da amostra. A avaliação ultrassonográfica evidenciou 42 casos de tireoidite. Os resultados apresentados confirmam a prevalência maior de nódulos de tireoide na população feminina com idade avançada. Entretanto, o achado de uma maior quantidade de nódulos de pequeno diâmetro com indicação de punção difere da literatura vigente.

\section{PALAVRAS-CHAVE}

Glândula Tireoide. Nódulo da Glândula Tireoide. Biópsia por Agulha Fina. 


\section{ABSTRACT}

The thyroid nodule is the presentation of various diseases of this gland. It is a very common clinical condition, particularly when employing sensitive methods of image. In this context, fine needle aspiration (FNA) is the main tool for differential diagnosis and the best value for money in the evaluation of nodular thyroid disease. This study aims to evaluate the profile of patients with thyroid nodules that are submitted to FNA. The sample consisted of 77 patients with thyroid nodule to be punctured. Data were collected for a period of 5 months in the Medical Center Sergipe Integrated (CEMISE), located in Aracaju / SE. The sample comprised 77 patients. Within the sample were 73 female patients $(94.8 \%)$ and 4 men (5.2\%), predominantly aged between 50 and 59 years. As the number of nodes, $57.1 \%$ of the patients (44 patients) had only one node to be punctured, while $42.9 \%$ (33 patients) had more

\section{RESUMEN}

El nódulo de tiroides es la presentación de diversas enfermedades de esta glándula. Se trata de una enfermedad muy común, especialmente cuando se emplean métodos de imágenes sensibles. En este contexto, la aspiración con aguja fina es la principal herramienta para el diagnóstico diferencial y el más rentable en la evaluación de la enfermedad tiroidea nodular. Este estudio tiene como objetivo evaluar el perfil de los pacientes con nódulos de tiroides que se someten a punción aspirativa por aguja fina. La muestra estuvo constituida por 77 pacientes que tenían nódulos de tiroides a ser puncionados. Los datos fueron recogidos durante un período de cinco meses, el Centro de Medicina Integral de Sergipe (CEMISE), situado en Aracaju / SE. La muestra fue de 77 pacientes. Dentro de la muestra había 73 pacientes del sexo femenino $(94,8 \%)$ y 4 hombres $(5,2 \%)$, predominantemente con edades comprendidas entre 50 y 59 años. En cuanto al número de nódulos, el $57,1 \%$ de los pacientes than one. The majority (58.3\%) of the nodules studied showed size $=1.0 \mathrm{~cm}$. The familial history of thyroid disease was found in 42 patients (54.5\%) and hypothyroidism personal history corresponded to $16.9 \%$ of the sample. The ultrasound examination showed 42 cases of thyroiditis. The results confirm the higher prevalence of thyroid nodules in the female population with advanced age. However, the finding of a greater number of nodes with small diameter puncture indication differs from existing literature.

\section{KEYWORDS}

Thyroid Gland. Nodule of the Thyroid Gland. Fine-needle Biopsy.

(44 pacientes) tenía sólo un nodo a ser puncionado, mientras que el 42,9\% (33 pacientes) tenía más de uno. La mayoría $(58,3 \%)$ de los nódulos estudiados mostró tamaño $\geq 1.0 \mathrm{~cm}$. Antecedentes familiares de enfermedad de la tiroides se encontró en 42 pacientes $(54,5 \%)$ y antecedentes personales de hipotiroidismo representó el $16.9 \%$ de la muestra. La ecografía mostró 42 casos de tiroiditis. Los resultados presentados confirman la mayor prevalencia de nódulos tiroideos en mujer con edad avanzada. Sin embargo, el hallazgo de un mayor número de nodos con indicación de punción de diámetro pequeño difiere de la literatura existente.

\section{PALABRAS CLAVE}

Glándula Tiroides. Los Nódulos de la Glándula Tiroides. Biopsia com Aguja Fina. 


\section{INTRODUÇ̃̃O}

O nódulo tireoidiano é a forma de apresentação de várias doenças desta glândula. Eles são muito comuns na população, sendo encontrados em até $7 \%$ das mulheres e $1 \%$ dos homens adultos por meio da palpação, em áreas suficientes de iodo, aumentando sua incidência se avaliados em mulheres idosas (VANDER et al., 1968; ROSÁRIO et al., 2013). No estudo populacional de Whickham, a incidência de nódulos em mulheres foi de 5,3\% (TUNBRIDGE et al., 1977). Entretanto, estudos com ultrassonografia (US) revelam uma prevalência bem maior, podendo chegar a $68 \%$ em alguns estudos (GUTH et al., 2009). A maioria dos nódulos tireoidianos encontrados é benigna, os malignos representam apenas $5 \%$ desses nódulos (BRANDER et al., 2000).

0 câncer de tireoide representa $2 \%$ a $5 \%$ do total de câncer em mulheres e menos de $2 \%$ nos homens. 0 tipo morfológico mais comum de câncer de tireoide é o adenocarcinoma papilífero, representando cerca de 50 a $80 \%$ dos casos. Seguido pelos adenocarcinomas foliculares, cerca de 10 a $40 \%$ e medulares com cerca de 5 a $15 \%$ (INCA, 2014).

A ampliação do uso dos métodos de imagem aumentou a frequência do diagnóstico incidental de nódulos tireoidianos. A ultrassonografia tem sido considerada, atualmente, um excelente exame na avaliação da glândula tireoide, sendo o método mais eficaz para avaliar o número, tamanho, localização, estrutura e ecogenicidade do nódulo (HEGEDUS, 2001; ROSÁRIO et al., 2013). Dessa forma, a punção aspirativa por agutha fina guiada pelo ultrassom (PAAF-US) possibilita a escolha da região do nódulo a ser puncionado quando se trata de nódulo misto ou com área de degeneração cística, diminuindo a porcentagem de material insuficiente ou inadequado ao diagnóstico. Além disso, permite selecionar os nódulos com características ultrassonográficas suspeitas em uma área multinodular, possibilitando o diagnóstico de câncer, ainda que as- sociado a um bócio coloide adenomatoso ou tireoidite de Hashimoto (HEGEDUS, 2001; CERATTI et al., 2012).

As recomendações da sociedade brasileira de endocrinologia e metabologia para indicação de PAAF de nódulos de tireoide se baseiam em suas dimensões e em suas características ultrassonográficas. As recomendações atuais para indicação da PAAF são as seguintes: nódulos maiores ou iguais a $5 \mathrm{~mm}$ em pacientes com alto risco clínico de malignidade (passado de exposição ionizante no pescoço, história pessoal ou familiar de câncer de tireóide ou nódulo incidentalmente detectado no FDG-PET) ou com características suspeitas à US (hipoecogenicidade, microcalcificações, margens irregulares, vascularização predominante central e encontro de linfonodos cervicais com características suspeitas); nódulos sólidos hipoecoicos com 10 ou mais $\mathrm{mm}$; nódulos iso ou hiperecoicos maiores ou iguais a $15 \mathrm{~mm}$; nódulo complexo ou espongiforme maior ou igual a $20 \mathrm{~mm}$; qualquer nódulo com aparente invasão extratireoidiana (ROSÁRIO et al., 2013).

A PAAF como método diagnóstico é rápida, de baixo custo, virtualmente ausente de complicações e tem excelente reprodutibilidade. Ela é considerada o teste de diagnóstico inicial para estabelecer se essas lesões são benignas ou malignas. De acordo com a American Thyroid Association a PAAF é o teste padrão-ouro para avaliação inicial de um nódulo de tireoide (GRAF, 2004; HAMBLETON; KANDIL, 2013). A sensibilidade e a especificidade da PAAF variam com a literatura, sendo de 87 a 92,8\% e de 62 a 100\%, respectivamente (PECCIN et al., 2003; UGURLU et al., 2008; RAHIMI et al., 2013).

Até o momento são escassas as pesquisas referentes ao perfil dos pacientes com nódulos tireoidianos na região em estudo. Sendo assim, o presente estudo objetivou avaliar o perfil dos pacientes com nódulos tireoidianos que são submetidos à PAAF. 


\section{MÉTODOS}

0 delineamento do estudo foi prospectivo. A população utilizada consistiu nos pacientes agendados para realizar o exame após a aprovação do Comitê de Ética em Pesquisa com Seres Humanos da Universidade Tiradentes - SE (CEP/UNIT nº 050512) e permissão da utilização do espaço físico do Centro de Medicina Integrada de Sergipe (CEMISE), localizado no Município de Aracaju-SE, em demanda espontânea do serviço de endocrinologia, de modo aleatório, por um período de cinco meses.

Foi redigido um Termo de Consentimento Livre e Esclarecido (TCLE) conforme as normas do Conselho Nacional de Saúde do Ministério da Saúde explicitadas na resolução 196/96. Foi esclarecido aos participantes da pesquisa que os mesmos poderiam se recusar a participar do estudo, não respondendo a alguma pergunta do questionário e que os dados obtidos seriam sigilosos.

O critério de inclusão consistiu em ter pelo menos um nódulo tireoidiano a ser puncionado e em aceitar participar da pesquisa. 0 critério de exclusão consistiu em não assinar o TCLE. Os portadores de nódulos de tireoide foram encaminhados para realizar o exame pelo profissional auxiliar, ficando a cargo da equipe do presente estudo a realização da PAAF.

A coleta de dados foi realizada em dois momentos. No primeiro foi realizada uma entrevista com o paciente, sendo questionada a idade, o sexo, a ocupação, a naturalidade, o histórico pessoal e familiar de doença tireoidiana e se faz uso de medicamento para tireoide ou medicação anticoagulante. A entrevista foi realizada pelo mesmo estudante de medicina treinado. No segundo momento os dados foram coletados durante a realização da PAAF-US, observando o tamanho do nódulo, a textura do parênquima tireoidiano e se existe tireoidite associada. A equipe que realizou o exame foi a mesma durante todo o estudo, sendo composta por um endocrinologista com treinamento para realização de PAAF e por um ultrassonografista com experiência e preparo para guiar o exame.

A técnica utilizada no procedimento da PAAF foi a punção por aspersão, utilizando agulha $0,70 \times 25$ $\mathrm{mm}$ conectada a uma seringa de $10 \mathrm{ml}$. Os pacientes utilizaram o Emla creme ${ }^{\circledR}$ na região anterior do pescoço 30 minutos antes do procedimento. 0 aparelho utilizado para guiar o exame foi da marca PHILLIPS, modelo ENVISION, HD 7 ou HD 15.

As variáveis numéricas foram testadas quanto à normalidade por meio do teste de Shapiro-Wilk. Valores com distribuição normal foram apresentados em média e desvio padrão (DP). Os dados categóricos são descritos pelas suas frequências absoluta e relativa. Para todas as análises, foi utilizado o programa SPSS ${ }^{\circledR}$ (Statistical Package for Social Sciences, versão 20.0).

\section{RESULTADOS}

0 estudo teve uma amostra de 77 pacientes submetidos à punção aspirativa por agulha fina guiada por ultrassonografia. Destes, 94,8\%, o correspondente a 73 pacientes eram mulheres, enquanto que $5,2 \%$ (4 pacientes) eram homens. A média de idade dos pacientes foi 52,99 anos ( $D P=16,27)$. A faixa etária que teve maior número de pacientes foi 50-59 anos com um total de 17 pacientes, seguidos pelas faixas etárias de 60-69 e 40-49 que tiveram 15 e 14 pacientes, respectivamente. A faixa etária dos jovens menores de 20 anos foi a que teve menor número de pacientes.

Quanto ao número de nódulos, $57,1 \%$ dos pacientes (44 pacientes) tinham apenas um nódulo com indicação para punção, enquanto que 42,9\% (33 pacientes) tinham mais de um nódulo para puncionar. A média de nódulos por pessoa foi 1,56 ( $D P=0,769)$.

Quando foi analisado o tamanho do nódulo tireoidiano, levou em consideração o maior diâmetro. A mé- 
dia de tamanho foi 1,36 (DP=0,79). 58,3\% da amostra tinha nódulo $\geq 1,0 \mathrm{~cm}$, sendo a maioria dos nódulos.

Em relação à história pessoal de hipotireoidismo, $16,9 \%$ da nossa amostra referiu ser portadora desta patologia e utilizar medicação diária para reposição hormonal, enquanto que $83,1 \%$ (64 pacientes) negou hipotireoidismo. Dos que referiam ser portadores da doença, $100 \%$ fazia uso regular da medicação.

Ao ser questionado em relação à história familiar em parentes de primeiro grau de qualquer doença tireoidiana, 47 pacientes $(61,1 \%)$ negaram qualquer história, enquanto que 30 (38,9\%) possuíam. Desses, $22(73,3 \%)$ citaram a doença nodular benigna. Seis pacientes referiram hipotireoidismo em parente de primeiro grau e 6 pacientes referiam história familiar em parente de primeiro grau de câncer de tireoide. Um paciente referiu que um parente de primeiro grau realizou tireoidectomia, porém não soube explicar por qual motivo. No estudo atual 42 pacientes $(54,5 \%)$ tinham tireoidite, achado encontrado acidentalmente no exame de US.

Além disso, 17,6\% dos pacientes encaminhados não tinham indicação de PAAF pelos critérios atuais da Sociedade Brasileira de Endocrinologia e Metabologia.

\section{DISCUSSÃO}

A glândula tireoide normal livre de nódulos ou de tireoidite apresenta ecogenicidade homogênea, sendo mais ecogênica que os músculos adjacentes ao exame ultrassonográfico, isso ocorre, pois as ondas sonoras atingem a interface entre células e colóide em ângulo reto e são refletidas de volta ao transdutor, sem dispersão, ocasionando a imagem homogênea e de ecogenicidade considerada normal (FRATES et al., 2005).

O estudo de Rahimi e outros autores (2013) também encontrou grande discrepância entre os sexos, com $97,9 \%$ dos pacientes submetidos à PAAF do sexo feminino. A média de idade encontrada nesse estudo foi de 39,8 anos (DP = 12). Um estudo conduzido na Turquia com 1004 pacientes encontrou 85,3\% da amostra do sexo feminino e uma média de idade de 48,41 (DP = 13,36) (UGURLU et al., 2008).

No Brasil, alguns estudos mostram o perfil do paciente com nódulo tireoidiano. Ceratti e outros autores (2012) conduziram um estudo em São Paulo e encontraram idade média de 52 anos (DP $=13,7$ ), dos quais $90,4 \%$ era do sexo feminino, o que foi semelhante ao perfil encontrado em nosso estudo. 0 estudo de Tomimori e outros autores (2004), também realizado em São Paulo, obteve um percentual de 90,5\% de pacientes do sexo feminino submetidas à PAAF, com idade média de 47,8 anos.

Furlanetto e outros autores (2000), no Rio Grande do Sul, avaliaram a prevalência de nódulos de tireoide por ultrassonografia em mulheres acima de 40 anos. Neste estudo, as mulheres sem nódulos de tireoide eram mais jovens, com 53,1 anos (DP $=10,7$ ), do que as que apresentaram nódulo tireoidiano, que tinham em média 58,2 (DP = 10,6). Houve também um significante aumento do número de nódulos com o aumento da idade. Um estudo em Santa Catarina com 265 pacientes que realizaram tanto a PAAF quanto o exame anatomopatológico, também houve predominância do sexo feminino (85\%) e a média de idade foi de 47,75 anos (DP = 14,93) (MENEGASSI et al., 2013).

A grande discrepância entre sexo feminino e masculino encontrado no nosso trabalho, também, foi encontrada em estudos realizados pelo mundo. A média de idade do nosso estudo foi levemente maior do que alguns dos estudos comparados que não estratificaram faixa etária, embora os estudos de São Paulo tenham mostrado média de idade semelhante. É sabido que mulheres têm maior prevalência de nódulos tireoidianos e que estas procuram mais os serviços de saúde do que os homens. Além disso, uma maior prevalência de nódulos também é encontrada em pessoas idosas (GRAF, 2004; ROSÁRIO et al., 2013). 
Prova disso são os dados do Instituto Nacional de Câncer José Alencar Gomes da Silva (INCA), em que são esperados no ano de 2014, para o Brasil, 1150 casos novos de câncer de tireoide para o sexo masculino e 8050 para o sexo feminino, com um risco estimado de 1,15 casos/100 mil homens e 7,91 casos/100 mil mulheres.

Nas mulheres, o câncer de tireoide é o quarto mais comum na região Sul, o sexto mais comum nas regiões sudeste, nordeste e norte e o nono mais comum na região centro-oeste (INCA, 2014). Mais de 70\% da mortalidade por câncer de tireoide ocorre em pessoas idosas. Entre adultos jovens, a mortalidade é baixa, sem diferença significativa entre homens e mulheres. Entre adultos maduros e idosos a mortalidade é crescente e com aumento do número de mortes para mulheres. Entretanto, observa-se que a mortalidade por esse câncer apresenta queda continuada na maioria das populações. Isto se deve, principalmente, à melhoria do tratamento (GUIMARÃES et al., 2013).

O aumento da prevalência de nódulos de tireoide e, consequentemente, de câncer de tireoide pode ser explicado pela detecção precoce por meio da US e da PAAF (BENTES; MARTINS, 2009). A sensibilidade da US para detecção de nódulos tireoidianos é de aproximadamente $95 \%$, superior a outros métodos como tomografia computadorizada e ressonância magnética (MAZEH et al., 2007). O objetivo desse exame é selecionar os nódulos que têm maior risco de malignidade, por meio da medição do tamanho e da identificação de achados preditivos ultrassonográficos para a PAAF. Dessa forma, a US deve ser solicitada em todo paciente com nódulo tireoidiano (ROSÁRIO et al., 2013).

Estudos na literatura mostram uma maior quantidade de paciente com múltiplos nódulos. No estudo de Urgulu e outros autores (2008), 70,3\% dos pacientes tinham múltiplos nódulos. Rahimi e outros autores (2013) tinham, de todos os nódulos examinados, 32 (22,2\%) nódulos únicos e 112 (77,8\%) eram múltiplos nódulos.
Semelhante ao nosso estudo, Furlanetto e outros autores (2000), diante de uma população de mulheres, identificou cerca de $50 \%$ das nodulações como maiores que $1 \mathrm{~cm}$. Enquanto que Rahimi e outros autores (2013) encontraram a maioria dos nódulos com tamanho superior a $1,5 \mathrm{~cm}(56,9 \%)$.

As chances de câncer de tireoide aumentam com o tamanho do nódulo, e o estudo de Smith-Blindman e outros autores (2013) mostrou que os nódulos $>2$ centímetros tinham um maior risco de câncer em comparação com os nódulos com menos de $1 \mathrm{~cm}$. 0 estudo de Rahimi e outros autores (2013) não obteve o mesmo desfecho, e concluiu que o tamanho do nódulo não pode eliminar a possibilidade de malignidade, sendo necessária investigação para nódulos de qualquer tamanho.

Urgulu e outros autores (2008) encontraram prevalência de hipotireoidismo de 9,6\%, de 12,8\% de hipertireoidismo e $77,6 \%$ não possuíam alteração hormonal da tireoide, sendo a única alteração a presença de nódulo, compatível com nosso estudo onde a maioria dos pacientes era eutireidiano e apenas $16,9 \%$ apresentava hipotireoidismo.

A tireoidite de Hashimoto é a causa mais comum de hipotireoidismo permanente em regiões sem deficiência de iodo, correspondendo a mais de $90 \%$ dos casos (YAMASHIRO et al., 2007). A tireoidite, baseado na etiopatogenia e quadro clínico, podem ser divididas em oito entidades: tireoidite de Hashimoto, tireoidite subaguda linfocítica, tireoidite subaguda granulomatosa (de Quervain), tireoidite pós-parto, tireoidite infecciosa, tireoidite medicamentosa, tireoidite actínica e tireoidite fibrosante de Riedel (YAMASHIRO et al., 2007). O presente estudo encontrou uma frequência de $54,5 \%$ de tireoidite à US.

Vale ressaltar que história de câncer de tireoide em parente de primeiro grau é um achado na história que sugere risco do nódulo tireoidiano. Além da história familiar, pode ser fator de risco para malig- 
nidade: sexo masculino, extremos de idade, história de radiação ionizante cervical, diagnóstico prévio de câncer de tireoide tratado com tireoidectomia parcial, história familiar (parente de primeiro grau) de câncer de tireoide, nódulo com rápido crescimento ou muito volumoso com sintomas compressivos, nódulo endurecido aderido a planos profundos e com linfonodos suspeitos presentes e presença de síndromes hereditárias como a neoplasia endócrina múltipla tipo 2 (NEM 2) (RAGO et al.,2010; ROSÁRIO et al., 2013).

\section{CONCLUSÃO}

A avaliação do perfil de pacientes submetidos à PAAF revela e caracteriza grupos de risco para desenvolvimento de patologias nodulares tireoidianas. Nesse contexto, os resultados apresentados pelo presente estudo confirmam a maior prevalência de nódulos tireoidianos na população feminina e idosa e de maior diâmetro, igual ou maior a $1,0 \mathrm{~cm}$, semelhante aos demais estudos. A amostra estudada apresentou uma maior quantidade de nódulos únicos com indicação de punção.

Um grande número dos pacientes encaminhados para realizar a PAAF não tinha indicação pelos critérios da sociedade brasileira de endocrinologia e metabologia. Tendo em vista os aspectos observados, o presente trabalho ampara a identificação de grupos de maior prevalência de patologias tireoidianas em uma população até então não estudada. Além disso, serve como orientação para trabalhos posteriores que avaliem a correlação entre os fatores demográficos e os achados citológicos preditivos de malignidade, com o intuito de discutir sobre a redução da quantidade de procedimentos desnecessários que produzem custos para os serviços e ansiedade para o paciente. Bem como na orientação dos profissionais sobre os critérios de indicação da PAAF.

\section{REFERÊNCIAS}

BENTES, R.O.L.; MARTINS, W.P. Ultra-sonografia para a biópsia de nódulos sólidos da tiróide. Experts in Ultrasound: Reviews and Perspectives, v.1, n.3, 2009, p.145-150.

BRANDER, A.E.; VIIKINKOSKI, V.P.; NICKELS, J.I.; KIVISAARI, L.M. Importance of thyroid abnormalities detected at US screening: a 5-year follow-up. Radiology, v.215, n.3, 2000, p.801-806.

CERATTI, S.; GIANNINI, P.; SOUZA, R.A.S.; JUNIOR, O.F.R. Ultrasound-guided fine-needle aspiration of thyroid nodules: assessment of the ideal number of punctures. Radiol Bras, v.45, n.3, 2012, p.145-148.

FRATES, M.C.; BENSON, C.B.; CHARBONEAU, J.W.; CIBAS, E.S.; CLARK, O.H.; COLEMAN, B.G.; et al. Management of thyroid nodules detected at US: Society of Radiologists in Ultrasound consensus conference statement. Radiology, v.237, n.3, 2005, p.794-800.

FURLANETTO, T.W.; PECCIN, S.; SCHNEIDER, M.A.O.; ZIMMER, A.S.; REIS, P.S.; GENRO, S.K.; et al. Prevalência de nódulos de tireóide detectados por ecografia em mulheres após os 40 anos. Rev Ass Med Brasil, v.46, n.4, 2000, p.331-334.

GRAF, H. Doença Nodular de tireoide. Arq Bras Endocrinol Metab, v.48, n.1, 2004, p.93-104.

GUIMARÃES, R.M.; MUZI, C.D.; PARREIRA, V.G.; SANTOS, R.D.; SAMPAIO, J.R.C. Evolução da mortalidade por câncer de tireoide em adultos no Brasil. Arq Bras Endocrinol Metab, v.57, n.7, 2013, p.538-544.

GUTH, S.; THEUNE, U.; ABERLE, J.; GALACH, A.; BAMBERGER, C.M. Very high prevalence of thyroid nodules detected by high frequency $(13 \mathrm{MHz})$ ultrasound examination. Eur J Clin Invest, v.39, n.8, 2009, p.699-706. 
HAMBLETON, C.; KANDIL, E. Appropriate and accurate diagnosis of thyroid nodules: a review of thyroid fine-needle aspiration. Int J Clin Exp Med, v.6, n.6, 2013, p.413-422.

\section{HEGEDUS, L. Thyroid ultrasound. Endocrinol Metab Clin North Am, v.30, 2001, p.339-360.}

INSTITUTO NACIONAL DE CÂNCER JOSÉ ALENCAR GOMES DA SILVA (INCA). Síntese de resultados e comentários: câncer de tireoide. Estimativa 2014: incidência de câncer no Brasil. Disponível em: <http://www.inca.gov. br/estimativa/2014>. Acesso em: 8 jun. 2014.

MAZEH, H.; BEGLAIBTER, N.; PRUS, D.; ARIEL, I.; FREUND, H.R. Cytohistologic correlation of thyroid nodules. Am J Surg, v.194, n.2, 2007, p.161-163.

MENEGASSI, J.; BORDIN, V.; OLIVEIRA, S.S. Acurácia diagnóstica entre punção aspirativa por agulha fina (PAAF) e exame anatomopatológico de tireoide. J Bras Patol Med Lab [online], v.49, n.4, 2013, p.288-293.

PECCIN, S.; FURLANETTO, T.W.; CASTRO, J.A.S.; FURTADO, A.P.A.; BRASIL, B.A.; CZEPIELEWSKI, M.A. Nódulos de tireóide: valor da ultra-sonografia e da biópsia por punção aspirativa no diagnóstico de câncer. Rev Assoc Med Bras, v.49, n.2, 2003, p.145-149.

RAGO, T.; FIORE, E.; SCUTARI, M.; SANTINI, F.; DI COSCIO, G.; ROMANI, R.; et al. Male sex, single nodularity, and young age are associated with the risk of finding a papillary thyroid cancer on fine-needle aspiration cytology in a large series of patients with nodular thyroid disease. Eur J Endocrinol, v.162, n.4, 2010, p.763-770.

RAHIMI, M.; FARSHCHIAN, N.; REZAEE, E.; SHAHEBRAHIMI, K.; MADANIS, $H$. To differentiate benign from malignant thyroid nodule comparison of sonography with FNAC findings. Pak J Med Sci, v.29, n.1, 2013, p.77-80.
ROSÁRIO, P.W.; WARD, L.S.; CARVALHO, G.A.; GRAF, H.; MACIEL, R.M.B.; MACIEL, L.M.Z.; et al. Nódulo tireoidiano e câncer diferenciado de tireoide: atualização do consenso brasileiro. Arq Bras Endocrinol Metab, v.57, n.4, 2013, p.240-264.

SMITH-BINDMAN, R.; LEBDA, P.; FELDSTEIN, V.A.; SELLAMI, D.; GOLDSTEIN, R.B.; BRASIC, N.; et al. Risk of Thyroid Cancer Based on Thyroid Ultrasound Imaging Characteristics: Results of a Population-Based Study. JAMA Intern Med, v.173, n.19, 2013, p.1788-1796.

TOMIMORI, E.K.; BISI, H.; MEDEIROS-NETO, G.; DE CAMARGO, R.Y.A. Avaliação ultra-sonográfica dos nódulos tireóideos: comparação com exame citológico e histopatológico. Arq Bras Endocrinol Metab, v.48, n.1, 2004, p.105-113.

TUNBRIDGE, W.M.; EVERED, D.C.; HALL, R.; APPLETON, D.; BREWIS, M.; CLARK, F.; et al. The spectrum of thyroid disease in a community: the Whickham survey. Clin Endocrinol (Oxf), v.7, n.6, 1977, p.481-493.

UGURLU, S.; CAGLAR, E.; YESIM, T.E.; TANRIKULU, E.; CAN, G.; KADIOGLU, P. Evaluation of thyroid nodules in Turkish population. Intern Med, v.47, n.4, 2008, p.205-209.

VANDER, J.B.; GASTON, E.A.; DAWBER, T.R. The significance of nontoxic thyroid nodules. Final report of a 15-year study of the incidence of thyroid malignancy. Ann Intern Med, v.69, 1968, p.537-540.

YAMASHIRO, I.; SAITO, O.C.; CHAMMAS, M.C.; CERRI, G.G. Achados ultra-sonográficos na tireoidite. Radiol Bras, v.40, n.2, 2007, p.75-79. 
2. Graduando de Medicina da Universidade Tiradentes.

E-mail:m.gutocruz@gmail.com

3. Graduando de Medicina da Universidade Tiradentes.

E-mail: larabaracho@hotmail.com

4. Graduando de Medicina da Universidade Tiradentes.

E-mail: mota.priscilla@hotmail.com

Avaliado em: 10 de Novembro de 2014

Aceito em: 15 de Novembro de 2014

5. Médica Endocrinologista. Professora da Universidade Tiradentes. Mestre em Ciências da saúde. E-mail: francielle_temer@uol.com.br 